\title{
Steric-effect-induced enhancement of electrical-double-layer overlapping phenomena
}

\author{
Siddhartha Das ${ }^{1}$ and Suman Chakraborty ${ }^{2}$ \\ ${ }^{1}$ Physics of Fluids Group, J. M. Burgers Centre for Fluid Dynamics, University of Twente, P.O. Box 217, \\ 7500 AE Enschede, The Netherlands \\ ${ }^{2}$ Department of Mechanical Engineering, Indian Institute of Technology, Kharagpur, Kharagpur-721302, India
}

(Received 3 March 2011; revised manuscript received 18 May 2011; published 20 July 2011)

\begin{abstract}
In this paper, we demonstrate that nontrivial interactions between steric effect and electrical-double-layer (EDL) overlap phenomena may augment the effective extent of EDL overlap in narrow fluidic confinements to a significant extent by virtue of rendering the channel centerline potential tending to the $\zeta$ potential in a limiting sense as the steric effect progressively intensifies. Such a behavior may result in a virtually uniform (undiminished) magnitude of the EDL potential across the entire channel height and may cause lowering of the total charge within the EDL.
\end{abstract}

DOI: 10.1103/PhysRevE.84.012501

PACS number(s): $82.45 . \mathrm{Gj}$

Recent advances toward understanding nanoscale electrochemical hydrodynamics have led to the development of several widely applicable miniaturized devices and systems tuned for practical applications [1-8]. The intricate dynamics within the surface-bound double layer of charge, popularly known as the electric-double layer (EDL) [9], often dictates the functionalities of these systems to an extremely significant extent. Theoretical electromechanical analysis of the EDL, on the other hand, has attracted great interest for close to half a century [10-12], and well known solutions exist for EDL charge distribution in a fluidic confinement, obtained under classical approximations, such as mean field assumption, assumption of nonoverlapping EDLs, assumption of EDL ions as point charges, etc.

A growing need to understand the electromechanics of systems with overlapping EDLs, and/or with very high ionic concentration, and/or with very large $\zeta$ potential, has necessitated the underlying analysis to be extended beyond the above-mentioned simplified assumptions, even within the framework of mean field approximations. For example, several recent investigations demonstrate remarkable influences of EDL overlap on nanoscale electrokinetic transport [13-21], and there has been considerable effort in the theoretical modeling of the concerned phenomena [22-33], obeying the governing principle of net charge electroneutrality of an isolated channel.

Interestingly, independent of the considerations of EDL overlap, there has been a continuous endeavor toward relaxing the treatment of ionic species as point charges by aptly taking the effects of their finite sizes into account. Such finite size effects of ions have been shown to have tremendous influence on different applications of nanoelectrokinetic phenomena [34-42]. Stern, in his celebrated work [43], introduced corrections to the Poisson-Boltzmann (PB) equation to account for the unphysical divergences of the Gouy-Chapman model of the double layer and indicated the volume constraints of ions in the electrolyte phase $[43,44]$. However, the first complete ion-size-effect-induced modified Poisson Boltzmann (mPB) model was developed by Bikerman [45]. Over the next several years, Bikerman's mPB equation was reformulated by Grimley and Mott [46], Grimley [47], Dutta and Bagchi [48], Bagchi [49,50], Dutta and Sengupta [51], Wicke and Eigen [52-54], Wiegel and Strating [55], Strating and Wiegel
[56,57], and Bohinc and co-workers [58,59]. For a flat double layer, Kilic et al. [60] used the lattice approach to obtain Bikerman's mPB equation with a simple composite diffuse layer model. Issues delineating the concerned consequences on the resultant diffuse layer capacitances were also addressed by some other researchers [44,60,61], in contrast with the predictions obtained using the Gouy-Chapman theory [62].

A significant step forward in the development of the effect of ion size in PB description was achieved by the use of the hard-sphere (HS) model for the ions, which Biesheuvel and van Soestbergen, in their pioneering paper [63], showed to be capable of addressing several inconsistencies and limitations in the lattice model. Their approach is distinct from the earlier approaches in the sense that, while Bikerman [45] assumed the local available volume to be the total volume minus the volume of all ions plus their hydration shell, and Sparnaay [64] replaced the volume of the hydrated ions by the excluded volume, Biesheuvel and van Soestbergen empirically included an extended Carnahan-Starling equation of state to describe HS interactions in EDLs containing ions of different sizes and charges. In this way, they attempted to overcome certain important limitations of the traditional lattice based models, which invariably underestimated the excluded volume effect as well as erroneously considered the size of a lattice as equivalent to the size of a solvent molecule and not the hydrated site. Their results were in excellent agreement with experimental findings [65], and their model was successfully extended to study the case of mixture of ions of different sizes [66-69]. However, none of the above studies have reported any analysis based on the simultaneous considerations of steric effects and EDL overlap phenomenon.

Here, we demonstrate that, by simultaneous considerations of finite ion-size effect and EDL overlap, one can remarkably have a completely geometric phenomenon (i.e., the EDL overlap induced by the physical presence of a confinement having a length scale below a threshold limit) influenced by a completely thermoelectrochemical transport phenomenon as governed by the ionic-size effects. In this perspective, it is important to pinpoint the distinctive fundamental contributions from our Brief Report as compared to some statistical mechanics based investigations (postulated on the density functional formalism) of Urbanija et al. [70] and Perutkova et al. [71] in 
which they have considered finite ion sizes and confinement simultaneously. In their studies, the ions considered are giant macroions that are randomly placed inside a confined channel. Minimization of the free energy of the system results in governing integrodifferential equations for the potential distribution within the confinement. Such an approach, however, may be intrinsically complex in addressing complicated scenarios, and in many cases, reduces analytical tractability so that nontrivial numerical treatment may be necessary to address even a simple problem [60]. Moreover, their approach never considers the condition of EDL overlap (realized when the confinement size goes below a threshold limit in relation to the Debye length so that a nonzero centerline potential is realized that cannot be prescribed a priori). In this context, it is important to mention that mere consideration of a confined geometry does not implicate the consideration of an EDL overlap phenomenon, since, if the characteristic length scale is beyond a critical limit (typically on the order of the Debye length), then far-stream boundary conditions on the electrostatic potential can effectively be employed at the channel centerline, rendering the situation qualitatively identical to the one with a single charged plate, despite the physical existence of a confinement.

The mathematical model employed in the present Brief Report follows from the modified space charge model developed by Cervera and co-researchers [72,73] in which the ionic-size effect is explicitly incorporated in the ionic flux density expression in the governing species transport equation as follows: $\vec{J}_{i}=\vec{u} n_{i}-D_{i} n_{i} \vec{\nabla}\left(\ln a_{i}+\frac{z_{i} F \phi}{R T}\right)$, where $\vec{u}$ is the advective velocity, $F$ is the Faraday constant, $z_{i}$ is the valency of the $i$ th ionic species, $T$ is the absolute temperature, $D_{i}$ is the diffusivity of the $i$ th ionic species, $n_{i}$ is the number density of the $i$ th ionic species, $\phi$ is the resultant electrical potential, and $a_{i}$ is the activity of the $i$ th ionic species and is expressed in terms of the ionic concentrations $n_{i}$ as $a_{i}=\frac{n_{i} / n_{r}}{1-v \sum_{k} n_{k} / n_{r}}$. Here, $n_{r}$ is the reference ionic number density, and $v$ is the partial molal volume of the ionic species (may alternatively be termed the steric factor). This model is applied to a geometrical confinement in which the characteristic EDL thickness is specifically chosen to be larger than the channel half height. The resulting EDL overlap phenomenon is assessed in terms of the difference between the centerline potential and the $\zeta$ potential, as a combined consequence of electrochemical, thermodynamic, and a pure geometrical artifact of the system configuration. In fact, the channel centerline potential is not a priori known in the present context, which needs to be calculated in consistency with the $\zeta$ potential and the potential gradient developed within the geometrical confinement as a function of the partial molal volume of the ionic species. Thus, the channel centerline potential derived in a self-consistent fashion, effectively represents the extent EDL overlap. The central result of our calculation is that the finite ion-size effects may remarkably enhance the effective extent of EDL overlap, quantitatively delineated by the proximity of the channel centerline potential with the $\zeta$ potential, as compared to the cases without involving finite ion-size effects. The resulting effective extent of the EDL overlap, under critical circumstances, may be so significantly enhanced that one may recover the $\zeta$ potential virtually undiminished across the entire channel height. This implies that one can completely ignore the EDL and can end up, quite dramatically, with an ideally homogeneously charged nanofluidic system.

For the present Brief Report, we consider a nanofluidic system (essentially a slit type nanochannel connected with its end reservoirs) in which the steady state transport phenomenon of each ionic species may be represented by the following [72,73]: $\vec{\nabla} \cdot \vec{J}_{i}=0$. In the absence of the bulk advective transport of ions, the same consideration implicates

$$
k_{B} T \ln \left(a_{i}\right)+e z_{i} \psi=\text { constant }
$$

where $\psi$ is the EDL potential, $e$ is the protonic charge, and $k_{B}$ is the Boltzmann constant. Considering equilibrium between the nanochannel and its connecting reservoirs (where $n_{+}=n_{-}=$ $n_{\infty}$ and $\psi=0$ ) and assuming a $z: z$ symmetric electrolyte for simplicity, one gets

$$
n_{ \pm}=\frac{n_{\infty}}{1+\frac{2 v n_{\infty}}{n_{r}}\left[\cosh \left(\frac{e z \psi}{k_{B} T}\right)-1\right]} \exp \left(\mp \frac{e z \psi}{k_{B} T}\right) .
$$

Substituting the above in the Poisson equation $\frac{d^{2} \psi}{d y^{2}}=$ $\frac{-\rho_{e}}{\varepsilon}=\frac{-e z\left(n_{+}-n_{-}\right)}{\varepsilon}$ (where $\varepsilon$ is the permittivity of the ionic $\stackrel{\varepsilon}{\varepsilon}$ edium and $y$ is the channel wall-normal coordinate) and integrating the same from the Stern layer-diffuse layer interface to the channel centerline (where $\frac{d \psi}{d y}=0$ and $\psi=\psi_{c} \neq$ 0 under overlapped EDL conditions), one gets

$$
\frac{d \psi}{d y}= \pm \frac{\psi_{r}}{\lambda \sqrt{v}} \sqrt{\ln \left\{\frac{1+\left[\cosh \left(\frac{\psi}{\psi_{r}}\right)-1\right]}{1+2 v\left[\cosh \left(\frac{\psi_{c}}{\psi_{r}}\right)-1\right]}\right\}}
$$

where $\lambda$ is the Debye length $\left(\frac{1}{\lambda^{2}}=\frac{2 n_{\infty} e^{2} z^{2}}{\varepsilon k_{B} T}\right)$ and $\psi_{r}=\frac{k_{B} T}{e z}$ is a reference potential.

In an effort to obtain the variations in $\psi$, we employ an iterative solution technique, where we start with a guessed value of $\psi_{c}$ and iteratively obtain a corrected value of the same by using the EDL potential gradient as given by Eq. (3) and a specified $\zeta$ potential, until convergence is achieved. It is important to mention here that instead of a given $\zeta$ potential boundary condition, one could as well employ a given surface charge density boundary condition or, more appropriately, a chemical equilibrium based interfacial treatment [74]. While the later ones effectively include more parameters into the iterative framework mentioned as above, the same does not introduce any essential new physics in the context of the physical issue addressed in this Brief Report. Accordingly, the specified $\zeta$ potential boundary condition is used in the present simulations for simplicity, without sacrificing the essential physics.

The central result of this Brief Report is the variation in the ratio $\psi_{\mathrm{c}} / \zeta$ with the partial molal volume or the effective steric factor $v$, for different $\lambda / H$ ratios (see Fig. 1). The effective extent of EDL overlap is essentially governed by the parameter $\psi_{\mathrm{c}} / \zeta$ (the closer its value to unity, the more intense the EDL overlap). From Fig. 1, it is evident that an increase in $v$ enhances $\psi_{\mathrm{c}} / \zeta$, implicating a greater extent 


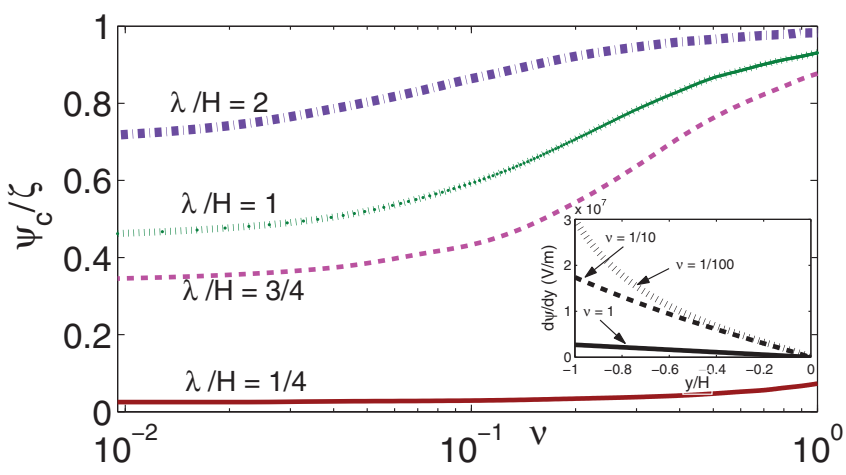

FIG. 1. (Color online) Variation of the ratio $\psi_{\mathrm{c}} / \zeta$ with steric factor $v$ for different $\lambda / H$ ratios. In the inset of the figure, we show the transverse variation of $d \psi / d y$ for the channel bottom half for $\lambda / H=$ 1 for different values of steric factor $\nu$. Results are provided for $H=$ $5 \mathrm{~nm}$ and $\zeta=-100 \mathrm{mV}$.

of EDL overlap (theoretically, the maximum extent of EDL overlap occurs when $\psi_{\mathrm{c}} / \zeta \rightarrow 1$ ). This enhancement is most prominent for channels where the EDL overlap phenomenon can be predicted even in the absence of steric effects. For such systems, realizable values of the steric factor ensure that a state is reached where $\psi_{\mathrm{c}} / \zeta \rightarrow 1$. Such a prominent influence of the steric effect in magnifying the extent of EDL overlap, however, is not witnessed for channels that have nonoverlapping EDLs in the absence of steric effects. To understand such a role of the steric effect, we study the variation of the transverse gradient of the EDL (see the inset of Fig. 1) as a function of $v$. Larger $v$ leads to a weaker EDL potential gradient [this is clearly noticeable from the analytical expression in Eq. (3)], causing a much weaker decay (with height) of the wall conditions and making it penetrate much deeper into the nanochannel bulk. Consequently, the channel centerline potential has a value significantly closer to the $\zeta$ potential, which, in effect, signifies a more enhanced EDL overlap. The manifestation of pronounced influence of the steric effect, based on the steric-effect-independent extent of EDL interactions, can be argued from steric-effect-driven weaker crowding of ions in the EDL. For an overlapped EDL even in the absence of steric effects, the EDL counterions are in a spars condition so that the average attractive pull of the charged wall is substantially weak. For such cases, the contribution of the entropic mixing of the ions (caused by their finite sizes, see Ref. [60]) can easily overcome the electrostatic attractive pull of the wall, ensuring a homogeneous mixing and, hence, a uniform distribution (rather than a wall-potential-dictated distribution) of the ions across the channel height. This effectively leads to a significantly enhanced value of the channel centerline potential, implying a high effective degree of EDL overlap. On the contrary, for an initially nonoverlapping system (even for finitely large EDL thicknesses), the ions are always

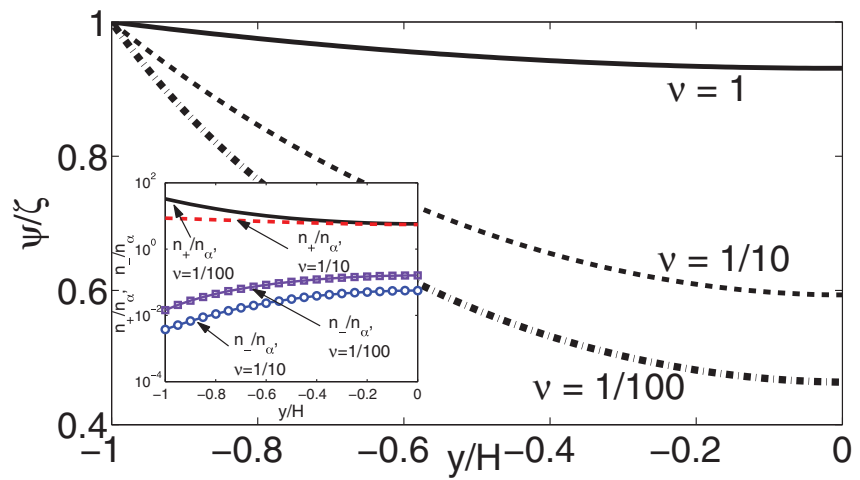

FIG. 2. (Color online) Transverse variation of the EDL potential $(\psi)$ for the channel bottom half, corresponding to $\lambda / H=1$ for different values of the steric factor $v$. In the inset of the figure, we show the corresponding transverse variation of the number density of the counterions $\left(n_{+} / n_{\alpha}\right)$ and coions $\left(n_{-} / n_{\alpha}\right)$. Results are provided for $H=5 \mathrm{~nm}$ and $\zeta=-100 \mathrm{mV}$.

tightly bound within the attractive range of the channel wall so that the entropic considerations can never lead to uniform distribution of the ions across the channel. Hence, for such cases, only minor enhancement of the extent of EDL overlap can be noted.

The consequences of such a steric-effect-induced enhancement of EDL overlap are remarkable. For example, the EDL potential, under such conditions, remains virtually undiminished across the entire channel (see Fig. 2). This implies that it is possible to achieve a uniformly charged nanochannel where the behavior at any location in the conduit is exactly identical to that interfacing the wall. Implicitly, this signifies that the role of the added ions toward dictating how the bulk sees and how the EDL screens the wall ceases to be important. This can straightaway be related to the fact that there is a significant lowering of the number of ions in the EDL, as a consequence of a lowering of the interfacial potential gradient (see the inset of Fig. 2).

To summarize, we have shown that the steric effect can substantially enhance the extent of effective EDL overlap in narrow confinements. These enhancements are most prominent for channels that have some degree of steric-effectindependent EDL overlap (i.e., EDL overlap under hypothetical conditions of the ionic species taken as point charges). Such a phenomenon may turn out to be immensely significant, since it leads to the paradigm of a homogeneously charged nanofluidic system, where the concept of strong near-wall gradients due to EDL phenomena virtually appears to be nonexistent. This, in turn, may affect several electrokinetic phenomena that depend on the extent of EDL interactions in nanochannels, such as streaming potential and energy transfer effects in narrow fluidic confinements [75-80], confinement-influenced macromolecular separation [81-83], and many others.
[1] A. Watson, Science 289, 850 (2000).

[2] S. K. Griffiths and R. H. Nilson, Anal. Chem. 78, 8134 (2006).

[3] F. H. J. van der Heyden, D. Stein, K. Besteman, S. G. Lemay, and C. Dekker, Phys. Rev. Lett. 96, 224502 (2006).
[4] L. Bocquet and E. Charlaix, Chem. Soc. Rev. 39, 1073 (2010).

[5] M. Napoli, J. C. T. Eijkel, and S. Pennathur, Lab Chip 10, 957 (2010). 
[6] W. Sparreboom, A. van den Berg, and J. C. T. Eijkel, Nat. Nanotechnol. 4, 713 (2009).

[7] W. Sparreboom, A. van den Berg, and J. C. T. Eijkel, New J. Phys. 12, 015004 (2010).

[8] P. Abgrall and N. T. Nguyen, Anal. Chem. 80, 2326 (2008),

[9] R. J. Hunter, Zeta Potential in Colloid Science: Principles and Applications (Academic, New York, 1981).

[10] D. Burgreen, and F. R. Nakache, J. Phys. Chem. 68, 1084 (1964).

[11] C. L. Rice and R. Whitehead, J. Phys. Chem. 69, 4017 (1965).

[12] S. Levine, J. R. Marriott, G. Neale, and N. Epstein, J. Colloid Interface Sci. 52, 136 (1975).

[13] H. Ban, B. Lin, and Z. Song, Biomicrofluidics 4, 014104 (2010).

[14] R. Chein, C. Liao, and H. Chen, Nanoscale Microscale Thermophys. Eng. 14, 75 (2010).

[15] J. Jamaati, H. Niazmand, and M. Renksizbulut, Int. J. Therm. Sci. 49, 1165 (2010).

[16] X. Wang and W. Hong, Phys. Rev. E 81, 041803 (2010).

[17] E. C. Yusko, R. An, and M. Mayer, ACS Nano 4, 477 (2010).

[18] M. L. Kovarik, K. Zhou, and S. C. Jacobson, J. Phys. Chem. 113, 15960 (2009).

[19] R. Chein, H. Chen, and C. Liao, J. Electroanal. Chem. 1, 630 (2009).

[20] S. Chakraborty and A. K. Srivastava, Langmuir 23, 12421 (2007).

[21] S. Talapatra and S. Chakraborty, Eur. J. Mech. B 27, 297 (2008).

[22] W. Qu and D. Li, J. Colloid Interface Sci. 224, 397 (2000).

[23] C. L. Ren, Y. D. Hu, D. Q. Li, and C. Werner, J. Adhes. 80, 831 (2004).

[24] A. T. Conlisk, J. McFerran, Z. Zheng, and D. Hansford, Anal. Chem. 74, 2139 (2002).

[25] Z. Zheng, D. J. Hansford, and A. T. Conlisk, Electrophoresis 24, 3006 (2003).

[26] S. Bhattacharyya, Z. Zheng, and A. T. Conlisk, J. Fluid Mech. 540, 247 (2005).

[27] D. Stein, M. Kruithof, and C. Dekker, Phys. Rev. Lett. 93, 035901 (2004).

[28] E. H. Kwak and E. F. Hasselbrink, J. Colloid Interface Sci. 284, 753 (2005).

[29] S. Kang and Y. K. Suh, J. Mech. Sci. Technol. 20, 2250 (2006).

[30] R. B. Schoch and P. Renaud, Appl. Phys. Lett. 86, 253111 (2005).

[31] R. B. Schoch, H. van Lintel, and P. Renaud, Phys. Fluids 17, 100604 (2005).

[32] F. Tessier and G. W. Slater, Electrophoresis 27, 686 (2006).

[33] F. Baldessari and J. G. Santiago, J. Colloid Interface Sci. 325, 526 (2008).

[34] A. Garai and S. Chakraborty, Electrophoresis 31, 843 (2010).

[35] C. K. Hua, I. S. Kang, K. H. Kang, and H. A. Stone, Phys. Rev. E 81, 036314 (2010).

[36] H. J. Zhao, J. Phys. Chem. C 114, 8389 (2010).

[37] A. S. Khair and T. M. Squires, J. Fluid Mech. 343, 640 (2009).

[38] B. D. Storey, L. R. Edwards, M. S. Kilic, and M. Z. Bazant, Phys. Rev. E 77, 036317 (2008).

[39] L. H. Olesen, M. Z. Bazant, and H. Bruus, Phys. Rev. E 82, 011501 (2010).

[40] M. Z. Bazant, M. S. Kilic, B. D. Storey, and A. Ajdari, Adv. Colloid Interface Sci. 152, 48 (2009).

[41] M. Z Bazant, M. S. Kilic, B. D. Storey, and A. Ajdari, New J. Phys. 11, 075016 (2009).
[42] M. Z. Bazant, B. D. Storey, and A. A. Kornyshev, Phys. Rev. Lett. 106, 046102 (2011).

[43] O. Stern, Z. Elektrochem. 30, 508 (1924).

[44] V. Freise, Z. Elektrochem. 56, 822 (1952).

[45] J. J. Bikerman, Philos. Mag. 33, 384 (1942).

[46] T. B. Grimley and N. F. Mott, Discuss. Faraday Soc. 1, 3 (1947).

[47] T. B. Grimley, Proc. R. Soc. London, Sec. A 201, 40 (1950).

[48] M. Dutta and S. N. Bagchi, Indian J. Phys. 24, 61 (1950).

[49] S. N. Bagchi, Indian J. Chem. Soc. 27, 199 (1950).

[50] S. N. Bagchi, Indian J. Chem. Soc. 27, 204 (1950).

[51] M. Dutta and M. Sengupta, Proc. Natl. Inst. Sci. India 20, 1 (1954).

[52] E. Wicke and M. Eigen, Naturwiss. 38, 453 (1951).

[53] E. Wicke and M. Eigen, Z. Elektrochem. 56, 551 (1952).

[54] E. Wicke and M. Eigen, J. Phys. Chem. 58, 702 (1954).

[55] F. W. Wiegel and P. Strating, Mod. Phys. Lett. B 7, 483 (1993).

[56] P. Strating and F. W. Wiegel, J. Phys. A 26, 3383 (1993).

[57] P. Strating and F. W. Wiegel, Physica A 193, 413 (1993).

[58] K. Bohinc, V. Kralj-Iglic, and A. Iglic, Electrochim. Acta 46, 3033 (2001).

[59] K. Bohinc, A. Iglic, T. Slivnik, and V. Kralj-Iglic, Bioelectrochemistry 57, 73 (2002).

[60] M. S. Kilic, M. Z. Bazant, and A. Ajdari, Phys. Rev. E 75, 021502 (2007).

[61] A. A. Kornyshev, J. Phys. Chem. B 111, 5545 (2007).

[62] D. L. Chapman, Philos. Mag. 25, 475 (1913).

[63] P. M. Biesheuvel and M. van Soestbergen, J. Colloid Interface Sci. 316, 490 (2007).

[64] M. J. Sparnaay, Recl. Trav. Chim. Pays-Bas 77, 872 (1954).

[65] V. L. Shapovalov and G. Brezesinski, J. Phys. Chem. B 110, 10032 (2006).

[66] S. Lamperski and C. W. Outhwaite, J. Colloid Interface Sci. 328, 458 (2008).

[67] T. Goel, C. N. Patra, S. K. Ghosh, and T. Mukherjee, J. Chem. Phys. 132, 194706 (2010).

[68] V. B. Chu, Y. Bai, J. Lipfert, D. Herschlag, and S. Doniach, Biophys. J. 93, 3202 (2007).

[69] J. G. Ibarra-Armenta, A. Martín-Molina, and M. Quesada-Pérez, Phys. Chem. Chem. Phys. 11, 309 (2009).

[70] J. Urbanija, K. Bohinc, A. Bellen, S. Maset, A. Iglic, V. KraljIglic, and P. B. S. Kumar, J. Chem. Phys. 129, 105101 (2008).

[71] S. Perutkova, M. Frank, K. Bohinc, G. Bobojevic, J. Zelko, B. Rozman, V. Kralj-Iglic, and A. Iglic, J. Membr. Biol. 236, 43 (2010).

[72] J. Cervera, V. García-Morales, and J Pellicer, J. Phys. Chem. B 107, 8300 (2003).

[73] J. Cervera, P. Ramirez, J. A. Manzanare, and S. Mafe, Microfluid. Nanofluid. 9, 41 (2010).

[74] M. Wang and A. Revil, J. Colloid Interface Sci. 343, 381 (2010).

[75] S. Chakraborty and S. Das, Phys. Rev. E 77, 037303 (2008).

[76] S. Das and S. Chakraborty, Langmuir 26, 11589 (2010).

[77] T. Das, S. Das, and S. Chakraborty, J. Chem. Phys. 130, 244904 (2009).

[78] M. Wang and Q. Kang, Microfluid. Nanofluid. 9, 181 (2010).

[79] C.-C. Chang and R.-J. Yang, Microfluid. Nanofluid. 9, 225 (2010)

[80] P. Goswami and S. Chakraborty, Langmuir 26, 581 (2010).

[81] S. Das and S. Chakraborty, Electrophoresis 29, 1115 (2008).

[82] S. Das and S. Chakraborty, Langmuir 24, 7704 (2008).

[83] S. Das and S. Chakraborty, Langmuir 25, 9863 (2009). 